Sādhanā Vol. 40, Part 3, May 2015, pp. 803-817. (C) Indian Academy of Sciences

\title{
Experimental investigation of separated shear layer from a leading edge subjected to various angles of attack with tail flap deflections
}

\author{
K ANAND and S SARKAR* \\ Department of Mechanical Engineering, Indian Institute of Technology Kanpur, \\ Kanpur - 208016, India \\ e-mail: subra@iitk.ac.in
}

MS received 12 July 2014; revised 18 December 2014; accepted 8 January 2015

\begin{abstract}
Shear layer development over a thick flat plate with a semi-circular leading edge is investigated for a range of angles of attack under different pressure gradients for a Reynolds number of $2.44 \times 10^{5}$ (based on chord and free-stream velocity). The characteristics of the separated shear layer are very well documented through a combination of surface pressure measurement and smoke flow visualization. The instability of the separated layer occurs because of enhanced receptivity of perturbations leading to the development of significant unsteadiness and three-dimensional motions in the second-half of the bubble. The onset of separation, transition and the point of reattachment are identified for varying angles of attack and pressure gradients imposed by tail flap deflections. The data concerning bubble length, laminar portion and reattachment points agree well with the literature.
\end{abstract}

Keywords. Separation bubble; transition; bubble bursting.

\section{Introduction}

A separation bubble occasionally forms on the suction surface of a highly cambered low pressure turbine blade or on the leading edge of an aerofoil at high angle of attack. A separated flow governs the development of the downstream boundary layer on the aerofoil and its stall characteristics apart from the reduction in lift and increase of drag. Since the separated shear layer is highly unstable, it undergoes rapid transition with amplification of background disturbances, changing the characteristics of downstream boundary layer. Also, a separated shear layer is highly sensitive towards surface roughness, free stream disturbances, pressure gradient, angles of attack and Reynolds number. Thus, it is important to understand the complex flow phenomenon to predict the onset of separation bubble including its transition, reattachment and further development for various conditions.

*For correspondence 
Extensive studies have been reported illustrating growth of a laminar separated layer leading to breakdown. Jones (1934) was the first to identify the existence of a laminar separation bubble on an aerofoil working under low Reynolds number. The flow downstream of separation was dictated by the nature of flow at the onset of separation. Roskho \& Lau (1965) carried out experiments on a separation bubble formed over a flat plate with various fore-body shapes at the leading edge. The bubble was characterized based on the surface pressure measurement. After a careful analysis of the results, a correlation was suggested based on the minimum and maximum pressure, downstream to separation. The results showed that the proposed correlation has a certain value at the point of reattachment and is found to depend highly on the flow geometry. A remarkable advancement in understanding the bubble structure and behaviour came with the work of Gaster (1967). He demonstrated the instability, breakdown apart from criterion for bubble bursting for combinations of pressure distribution and Re. Horton (1968) proposed a semi-empirical model describing growth and bursting of bubbles. It should be noted that a laminar separation bubble occasionally forms on aerofoils and stalling is ultimately determined by bursting, where reattachment may occur far downstream or may not occur at all. Crabtree (1969) made an attempt to find a universal value for predicting the bursting of short bubbles based on the pressure data available at separation and reattachment points. He predicted that there is a maximum possible value for the pressure recovery factor for which a short bubble can sustain over the range of Reynolds number investigated. Also, they found that the breakdown of short bubble appears to be a strong function of angle of incidence and Reynolds number. Tani (1964) presented a review paper from the published experimental results for varied geometrical sections (aerofoils, cylinders) that involve flow separation. He suggested that separation is evident only when the Reynolds number based on boundary layer displacement thickness at separation is greater than a critical value. The behaviour of the separation bubble is very well documented by Arena \& Mueller (1980) for various angles of attack combined with tail flap deflections and for a range of Reynolds number. The separation bubble was characterized with smoke flow visualization and surface pressure measurements. Their results showed that the bubble dimensions are very much susceptible to angles of attack and Reynolds numbers.

Many experimental studies (Gerakopulos et al 2010; Boutilier \& Yarusevych 2012; O'Meara \& Mueller 1987; Samson et al 2012; Wahidi \& Bridges 2012) demonstrated the onset of separation, transition and the point of reattachment with pressure measurements and complemented their results with flow measurements. Extensive pressure measurements (Cherry et al 1983; Yarusevych et al 2008) were carried out on the separated zone to reveal the unsteady nature of the separated shear layer. Pressure measurements are complemented with flow visualizations. A low frequency motion associated with the flapping of shear layer attributing to the change in bubble length was reported. Recently, Hu \& Yang (2008) performed experiments on a NASA low-speed aerofoil to characterize the unsteady behaviour of the laminar separation bubble with a high resolution PIV for various angles of attack. Reynolds stresses obtained from PIV measurements showed a significant increase in magnitude, downstream to the laminar part of the separation bubble and the critical value 0.001 indicates the beginning of transition process. Hain et al (2009) and McAuliffe \& Yaras (2005) used PIV measurements to investigate a laminar separation bubble on an aerofoil. The study revealed the unsteady flow structures and their significance in the production of turbulence for various angles of attack. Their results showed the presence of Kelvin-Helmholtz $(K-H)$ rolls in the separated shear layer which rapidly amplify leading to three-dimensional breakdown to turbulence.

Numerical investigations (Lin \& Pauley 1996) were made on a separated flow over an aerofoil for various angles of attack. Their results showed a periodic vortex shedding and subsequent pairing of vortex in the vicinity of reattachment zone. Also, it was reported that the onset of 
separation shifts with change in angles of attack. Yang \& Voke (2001) carried out LES to demonstrate the features of separated shear layer over a semi-circular leading edge and showed that the instability occurred via $K-H$ mechanism. Their instantaneous results showed that the reattachment locations are found to vary over $53 \%$ of the mean reattachment length. In turbomachinery applications, a separation bubble occasionally forms on the suction surface of a LPT and the flow becomes highly unsteady due to the periodic wake passing. The excitation of such a separated layer by periodic wakes was carried out by Sarkar $(2007,2008)$. The LES revealed the formation of coherent vortices by the rollup of the shear layer. The transition during the wake-induced path was governed by a mechanism that involves the formation and convection of these vortices followed by the production of turbulent kinetic energy.

In this paper, the characteristics of a laminar separation bubble from the semi-circular leadingedge of constant thickness aerofoil model has been documented for angles of attack $(\alpha)$ in the range $0^{\circ}-10^{\circ}$ combined with flap angles $(\beta)$ of $0^{\circ}$ and $\pm 30^{\circ}$, following the work of Arena \& Mueller (1980). However, smoke flow visualisation by Arena and Mueller to explain the dynamics of a separation bubble remains qualitative. In the present work, in addition to the surface pressure measurements, the flow visualisations are quantified with PIV illustrating the instantaneous variation in the reattachment position. The purpose of the investigation is to identify the onset of separation, transition, the point of reattachment and the limiting value for short bubble sustenance. An insight of laminar separation bubble is also discussed. The data presented are of fundamental importance to the gas turbine community as flow separation often occurs in gas turbine blades apart from low-Reynolds number aerofoil.

\section{Experimental setup}

Experiments are carried out in a blowing wind tunnel having an average $\mathrm{Tu}$ of $1.2 \%$ that can achieve velocities in the range of 3-15 m/s. The blower at the upstream end of the tunnel is powered by a three phase induction motor. The exit of the blower is followed by a diverging portion that extends to a length of $0.305 \mathrm{~m}$. A flow conditioning chamber follows the end of diverging section that houses a honeycomb and three anti-turbulence screens leading to a nozzle. The nozzle is designed for a two-dimensional contraction of 6:1 area ratio which leads to the test section. The cross-section of the test section measures $0.610 \mathrm{~m} \times 0.230 \mathrm{~m}$ and extends for a length of $0.750 \mathrm{~m}$. A schematic of the tunnel with the model mounted in the test section is shown in figure 1. The aerofoil is modelled as a flat plate with semicircular leading edge along with tail-flap hinged at the downstream end of flat portion. The leading edge diameter $(D)$ is $0.10 \mathrm{~m}$ in size and the entire model is firmly held between the side walls of the test section. The flat portion of the model from the leading edge of the blend point measures $4 D$ in length. The tailflap of $2 D$ length can be moved on either side to a maximum of $45^{\circ}$ about its hinge and serves to impose a pressure gradient. Static taps, 59 in numbers, each of $0.8 \mathrm{~mm}$ hole diameter are drilled at its midspan and are distributed along the entire length of the upper surface, beginning from the lower end of the leading edge.

Surface static pressures are acquired through Electronically Scanned Pressure (ESP) connected to the static taps distributed over the model through a PXI based data acquisition system. The ESP has a differential pressure range of $\pm 2.5 \mathrm{kPa}$ with a sampling rate of $20 \mathrm{kHz}$. The ESP has 64 ports and each port has an individual pressure sensing transducer that produces a voltage output. These pressure sensors have to be calibrated individually by applying a known pressure. The voltage obtained from the sensor of each port is converted into pressure through a second 


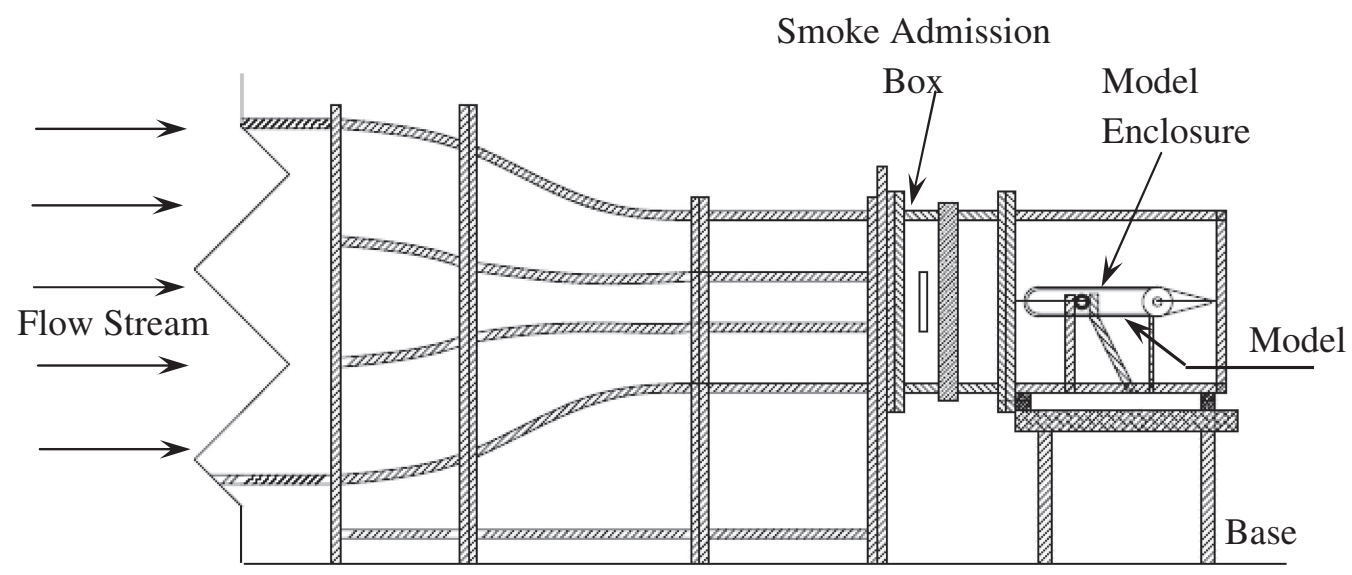

Figure 1. Schematic representation of the model mounted in the tunnel.

degree polynomial equation. Besides there are two additional reference ports meant for calibration and data acquisition. The electronic data are acquired through a PXI module that houses a DAQ card, NI-6132 with 4 channels, 14-bit configuration having a sampling rate of $2.5 \mathrm{MS} / \mathrm{s}$ and is connected to the computer through a NI card, PCIe-8361. An interface connects the ESP and the PXI module to enable data multiplexing. These instruments are powered by $\pm 12 \mathrm{~V}$ DC supply system and a $+5 \mathrm{~V}$ DC supply to excite the pressure sensors. The tunnel static is referenced to measure differential pressures and is considered for whole of the experiment. These pressure scanners are controlled by programmed software through LabVIEW. Accuracy of the instrument is maintained within $0.05 \%$ of the full scale pressure range through periodic calibration.

Smoke flow visualizations are carried out using a Dantec Flowsense camera capable of capturing $8 \mathrm{Fps}$ at a resolution of $2048 \times 2048$ pixels. To carry out the optical flow visualization, seeding particles of 3-5 $\mu \mathrm{m}$ size are admitted inside the tunnel; they glitter while they pass through a thin light sheet. The seeding particles are generated from a fogger fluid through a fog generating machine and a laser of $136 \mathrm{~mJ}$ capacity produces the required light sheet optics that is used in conjunction with the camera to enable the flow visualization.

\section{Results and discussion}

The experiments are conducted on the model for angles of attack $0^{\circ}-10^{\circ}$ with tail flap deflections of $0^{\circ}$ and $\pm 30^{\circ}$ at a $R e_{c}$ of $2.44 \times 10^{5}$ to illustrate the laminar separated bubble under different conditions. Pressure data are obtained from the static taps flushed out on the model surface. A schematic of the model is shown in figure 2(a). The measurements are made with reference to the origin located at the theoretical stagnation point of the model leading edge. It should be noted that the $x$-axis is referenced along the model chord line and the $y$-axis remains perpendicular to the model surface all through the experiments. Figure 2(b) and 2(c) shows the sign convention considered for the deflection of flap. A positive sign is associated when the flap is deflected down and a negative sign while the flap is deflected up. A flap deflection will impose a pressure gradient on the separated shear layer.

Surface pressures are measured from the static taps distributed along the midspan of the model using an ESP unit. The ESP is controlled through a Labview programme for data acquisition. The stream velocity at inlet of the test section is measured with a pitot tube considering tunnel 
(a)

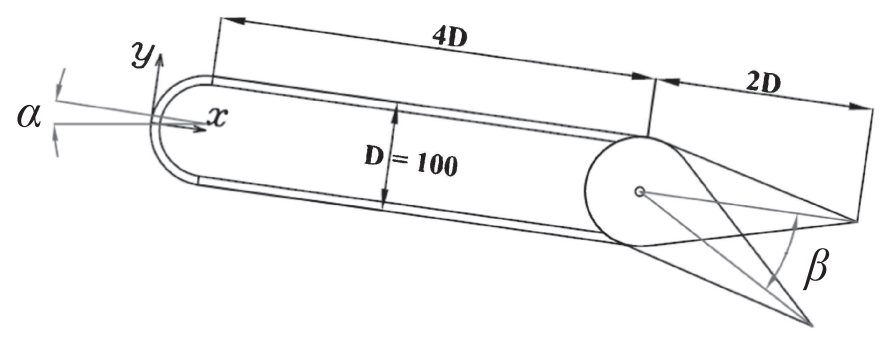

(b)

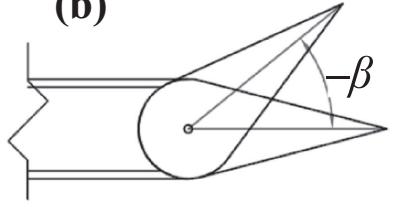

(c)

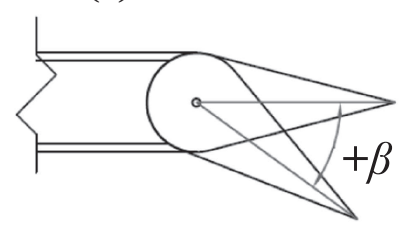

Figure 2. (a) Schematic representation of the model; (b) and (c) sign convention associated with the flap deflection.

static as reference. The pitot probe is held $1 D$ upstream of the model leading edge and of $1.5 \mathrm{D}$ above the model top surface. The dynamic pressure obtained from the pitot is used to normalize the surface static pressures. The surface pressure variations along the chord are nondimensionalised as $C_{p}^{\prime}=\left(C_{p}-C_{p \min }\right) /\left(C_{p \max }-C_{p \min }\right)$ following Roskho \& Lau (1965), which is called as pressure recovery coefficient. Figure 3 shows $C_{p}^{\prime}$ plotted against surface length non-dimensionalised with leading edge diameter for $\beta=0^{\circ}$ and $\alpha=0^{\circ}$. It is observed that the maximum pressure for $\beta=0^{\circ}$ and $\alpha=0^{\circ}$ occurs almost at the theoretical stagnation point i.e., at $S / D=0$, which is shown with a dotted line. Further, it illustrates that the flow undergoes acceleration in the downstream of stagnation point and attains a minimum value of $C_{p}^{\prime}$ depicting a suction peak at $S / D=0.61$. In the downstream of the suction peak, flow advances against

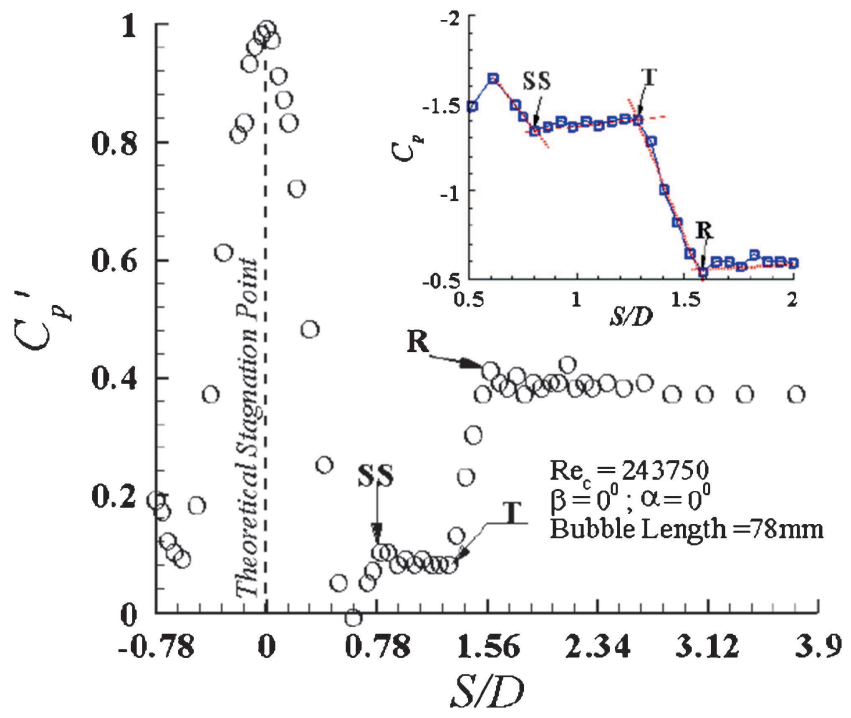

Figure 3. Pressure recovery coefficient plotted against the surface length non-dimensionalised with the leading edge diameter for zero angle of attack subject to zero tail flap deflection. SS - Separation, $\mathbf{T}$ - Transition and $\mathbf{R}$ - Reattachment. 
adverse pressure gradient for a short distance with a subsequent constant pressure plateau starting at $S / D=0.8$. Following the end of pressure plateau at $S / D=1.28$, there exists a region of strong adverse pressure gradient with a peak at $S / D=1.58$ that droops down slowly downstream. Previous studies suggest that the beginning of pressure plateau can be identified as the onset of separation (Arena \& Mueller 1980; Gaster 1967; McAuliffe \& Yaras 2005; Samson et al 2012; Cherry et al 1983), whereas the peak in the adverse pressure gradient region is identified as reattachment (Gerakopulos et al 2010; Hu \& Yang 2008; McAuliffe \& Yaras 2005). In detail, the onset of separation, transition and reattachment are estimated by following the procedure adopted by Gerakopulos et al (2010). A best fit line is drawn for the surface pressure data in the regions that is just upstream to the pressure plateau, the pressure plateau, the rapid pressure recovery region and downstream to the point of peak pressure. The point of intersection of these lines marks the locations of separation, transition and reattachment which is shown in the zoomed view of figure 3. The onset of transition and reattachment points are denoted as $T$ and $R$, whereas separation point by $S S$. It is observed that separation bubble formed on the surface of an aerofoil has two distinct regions: (1) a laminar portion, in the regions of constant pressure and (2) a region of rapid pressure rise due to transition to turbulence attributing to high momentum exchange from the free stream.

Figure 4 illustrates the growth of the separated shear layer for different angles of attack at zero flap angle deflection. The onset of separation shifts upstream with a delayed reattachment as angle of attack increases. Further, a slow increase of pressure is observed in the recovery region depicting prolonged transitional length with increasing $\alpha$. It is to be noted that the shear layer reattaches at far downstream leading to the formation of a larger separation bubble for $\alpha=7^{\circ}$ and the separated shear layer fails to reattach due to insufficient energy attributing to bubble bursting for $\alpha=10^{\circ}$. As expected, the maximum pressure near the leading edge shifts upstream to the theoretical stagnation point with increase of $\alpha$ and is clearly evident from the zoomed view. Previous experimental (Arena \& Mueller 1980; Gerakopulos et al 2010; Hu \& Yang 2008;

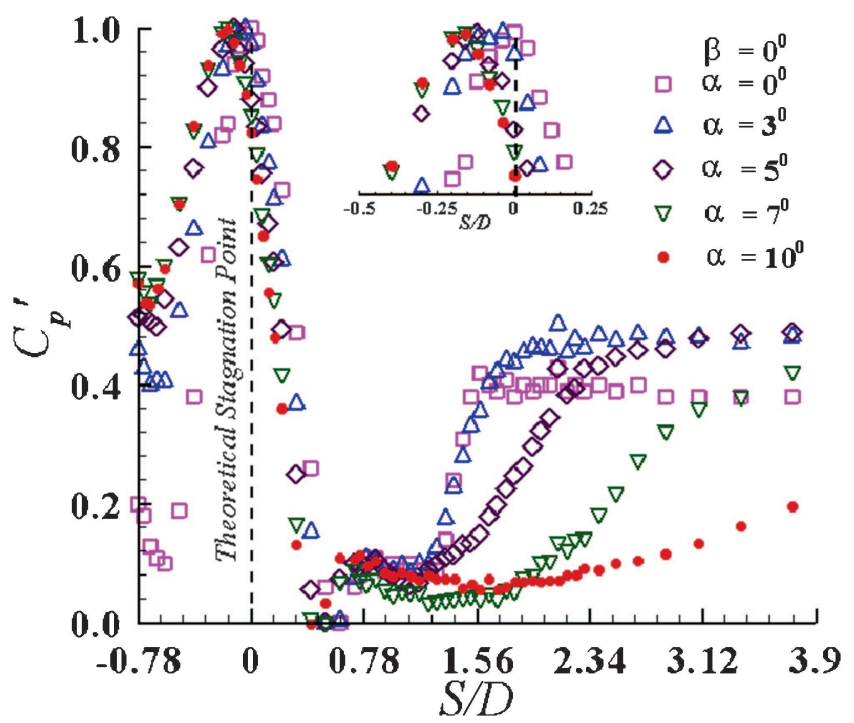

Figure 4. Growth of the separated shear layer for various angles of attack subject to zero flap deflection. 
Roskho \& Lau 1965) and numerical (Lin \& Pauley 1996) studies also illustrate a change in the onset of separation and reattachment with change in angles of attack.

Figure 5 shows the comparison of present experimental results with data obtained by Arena \& Mueller (1980) on a similar configuration. Here $C_{p}$ is plotted against the non-dimensional chord length. The angle of attack considered in the present study is $\alpha=5^{\circ}$ at a $\operatorname{Re}_{c}$ of $2.44 \times 10^{5}$, whereas $\alpha$ was $4^{\circ}$ at a $R e_{c}$ of $2.74 \times 10^{5}$ for Arena and Mueller. The present data agree well with those of Arena and Mueller illustrating the same trend from the onset of transition, however, a difference is observed in the dead air region. The possible reason for this difference is either with the angle of attack or the Reynolds number. The comparison also supports an initial laminar region exhibiting a pressure plateau that extends for a short distance downstream of separation and then curves up with a rise in $C_{p}$ due to transitional flow.

Figure 6 illustrates the effect of pressure gradient on the separated shear layer by changing the flap angles. Figure 6(a) illustrates the evolution of surface pressure on the model at $\alpha=0^{\circ}$ for different values of $\beta=0^{\circ},-30^{\circ}$ and $+30^{\circ}$. The trend of pressure in the recovery region is similar to all the cases. However, for $\beta=+30^{\circ}$, the reattachment is delayed with a higher pressure peak compared to $\beta=0^{\circ}$ and $-30^{\circ}$. The zoomed view shows that stagnation point shifts upstream for $\beta=+30^{\circ}$, whereas it shifts downstream for $\beta=-30^{\circ}$ with respect to theoretical stagnation point. For the case of $\alpha=3^{\circ}$ shown in figure $6(\mathrm{~b})$, with the tail flap deflected down by $30^{\circ}$ (for $\beta=+30^{\circ}$ ), the shear layer appears to be affected considerably with a slower pressure recovery attributing to an elongated transition length and a delayed reattachment. The onset of separation and length of transition are almost identical for $\beta=0^{\circ}$ and $-30^{\circ}$ as seen earlier. Further, the stagnation point moves upstream for all the cases of $\beta$.

It has been already stated that the tail flap deflection develops a pressure gradient on the separated layer. To quantify the imposed pressure gradient the variation of $C_{p}$ is presented in figure 7 for $\alpha=3^{\circ}$ and $\beta=0^{\circ}, \pm 30^{\circ}$ from the onset of separation. It is evident from the figure that the effect of pressure gradient is felt in the flow reversal region. Thus the pressure gradient

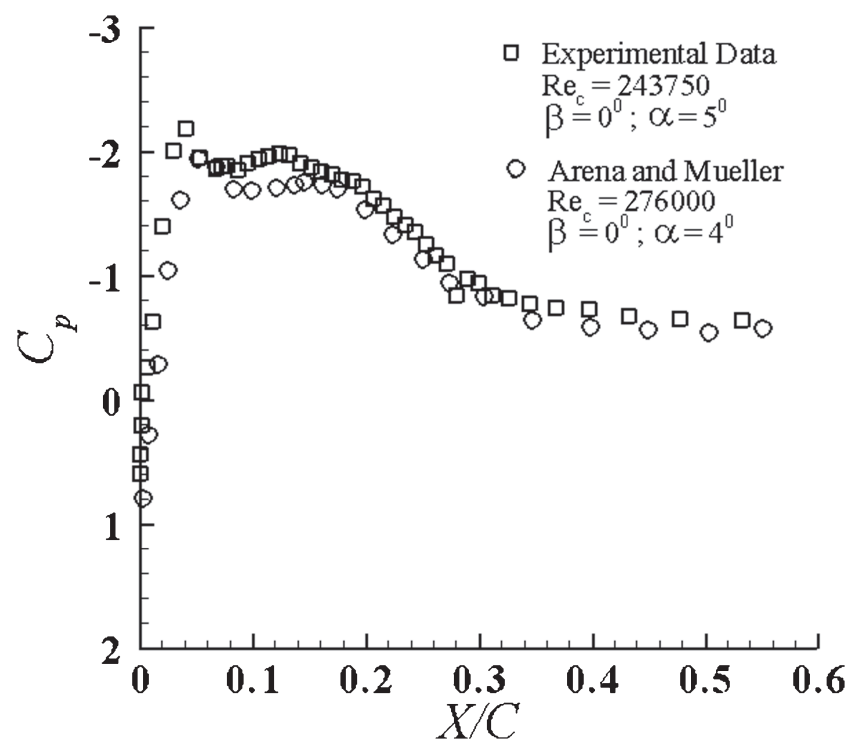

Figure 5. Comparison of experimental data with the data obtained from a similar model configuration by Arena and Mueller. 


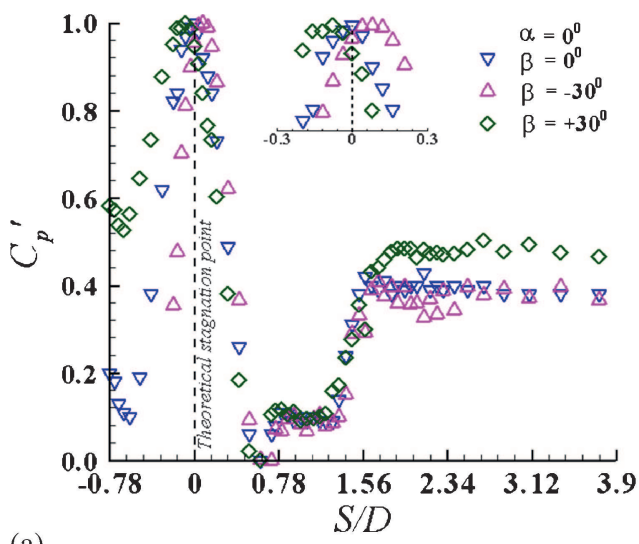

(a)

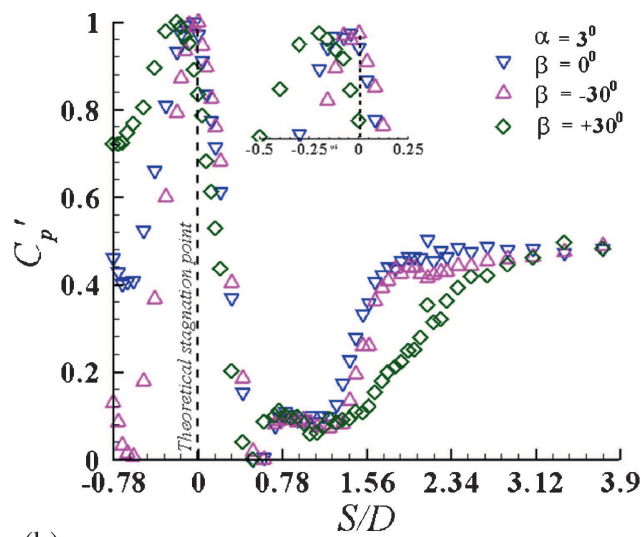

(b)

Figure 6. Effect of $\alpha$ on the growth of shear layer for various imposed pressure gradients: (a) $\alpha=0^{\circ}$ and (b) $\alpha=3^{\circ}$.

is quantified here by the best fit line drawn for the surface pressure through the transition region. The pressure gradient is found to be $16 \%$ higher for $\beta=-30^{\circ}$ and $59 \%$ lower for $\beta=+30^{\circ}$ with respect to $\beta=0^{\circ}$. In all these three cases, the pressure gradient in the transitional region is adverse. Thus, the delayed reattachment for $\beta=+30^{\circ}$ is due to relaxed adverse pressure gradient as compared to $\beta=0^{\circ}$ and $-30^{\circ}$.

The onset of separation, transition and reattachment point can be best identified from spatial growth rate of $C_{p}$ and are illustrated through figure 8. Upstream to separation, the growth of

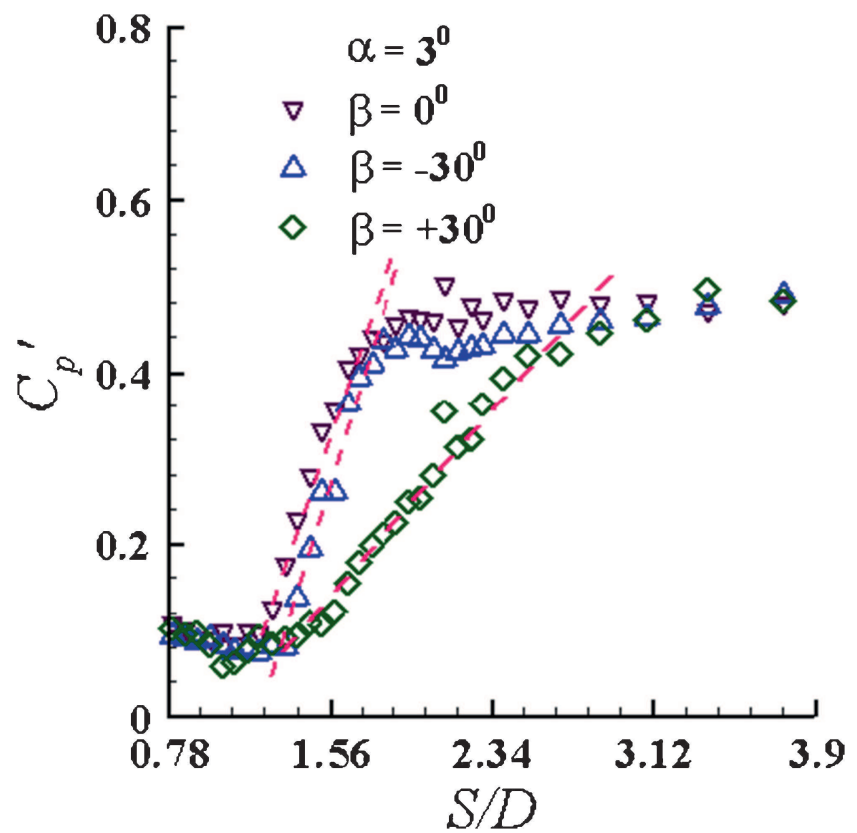

Figure 7. Effect of pressure gradient on the growth of shear layer detailed from the onset of separation to reattachment point for $\alpha=3^{\circ}$. 


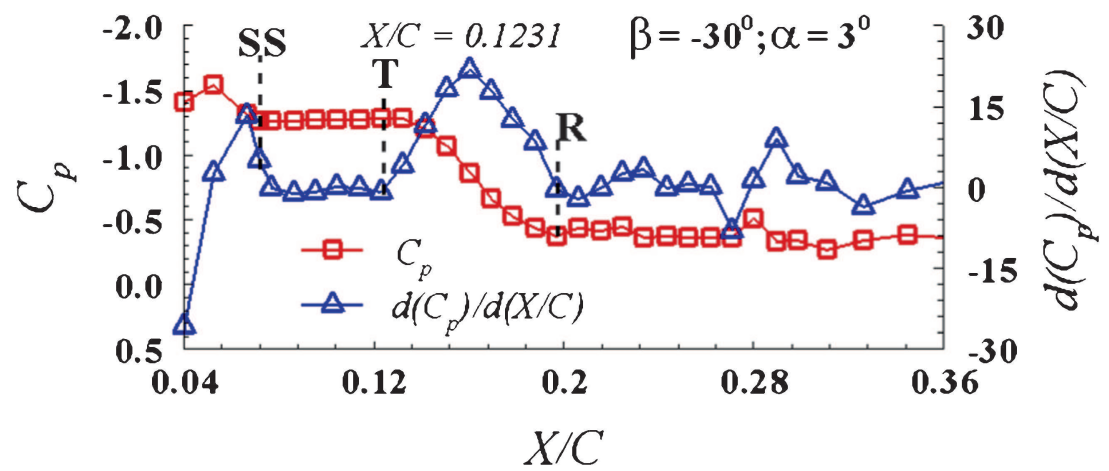

Figure 8. Identification of onset of separation, transition and reattachment from the spatial growth rate of $C_{p}$.

pressure gradient remains positive. The onset of separation is recognised with a sharp drop in growth rate of $C_{p}$ with a negative slope. A plateau that follows the negative slope with a zero growth rate in $C_{p}$ represents the dead air region. The onset of transition is identified at the downstream end of the plateau, where the growth rate of $C_{p}$ is just peaking up with a sudden change in slope. Similarly, reattachment occurs in the region of zero pressure gradient with a change in slope. The growth rate of $C_{p}$ becomes positive during transition, whereas it remains negative downstream to separation until it reaches the beginning of plateau. The shear layer exhibits a laminar nature between downstream to separation and the end of the plateau. The bubble length, onset of separation and transition measured from the growth rate of $C_{p}$ will be discussed in the following section.

Figure 9 shows the change in the onset of separation and transition apart from reattachment point of the separated shear layer for increasing angle of attack and different imposed pressure

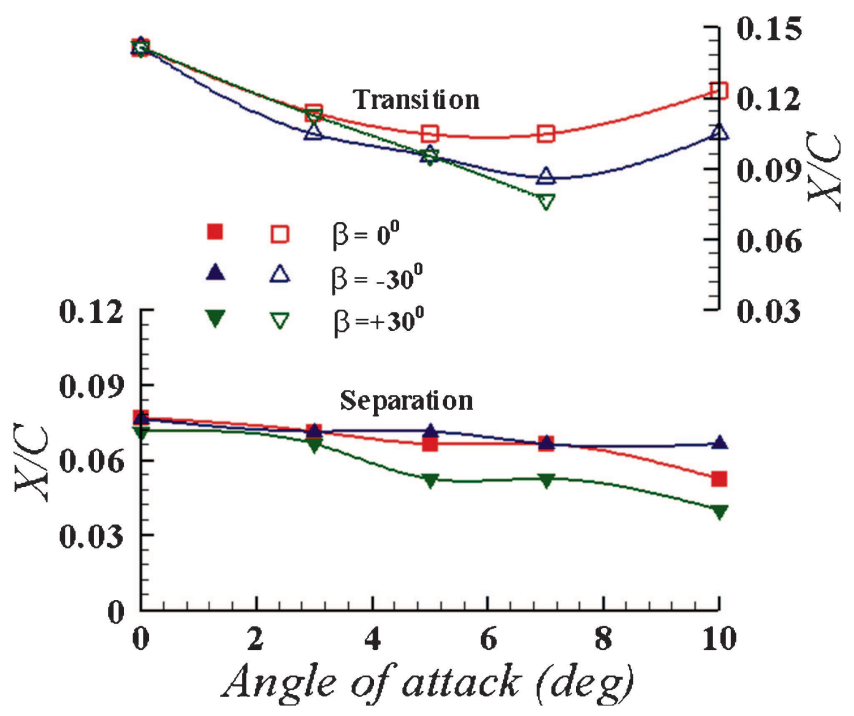

Figure 9. Effect of $\alpha$ and $\beta$ on the onset of separation and transition locations from the pressure measurement. 


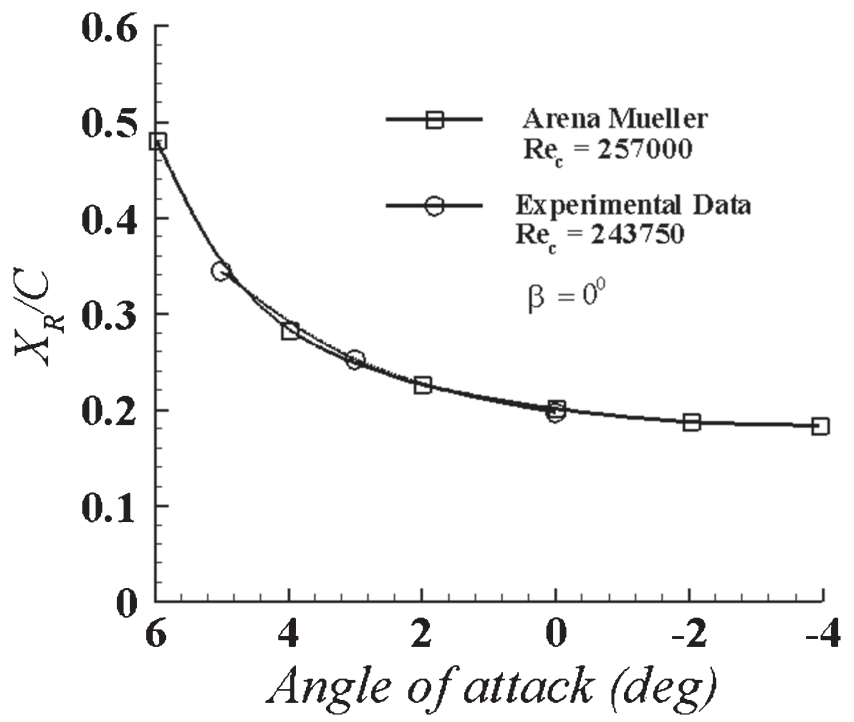

Figure 10. Comparison of reattachment locations plotted as a function of $\alpha$ with $\beta=0^{\circ}$ based on chord Reynolds number.

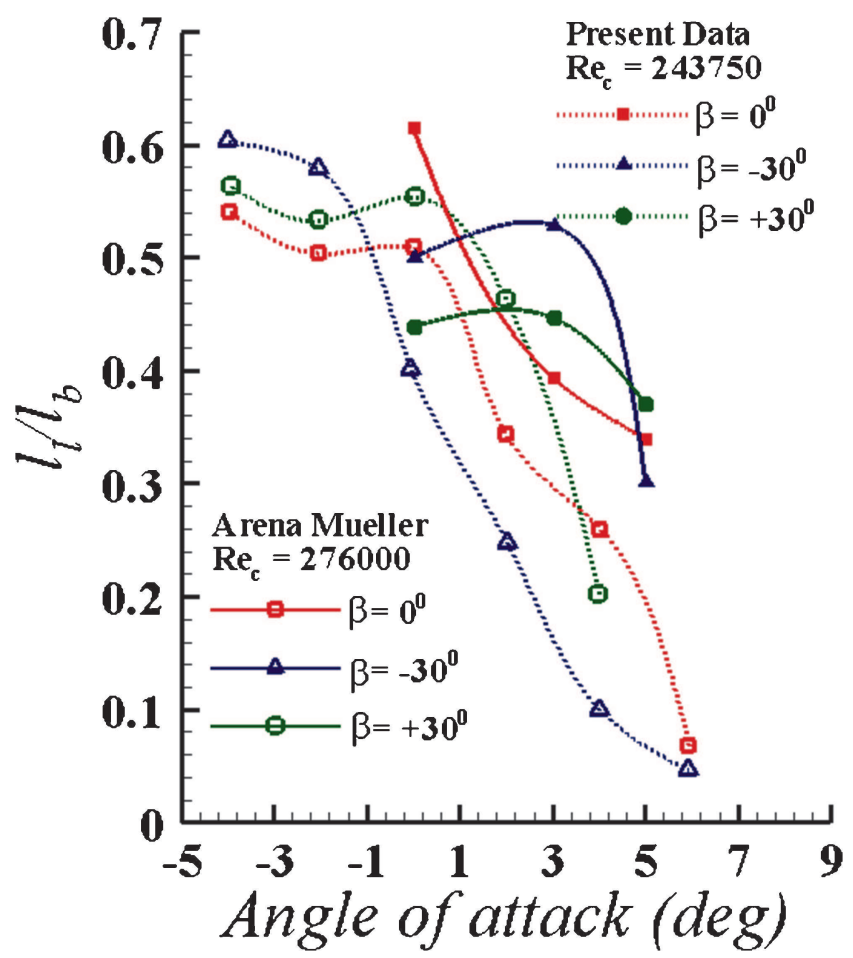

Figure 11. Laminar shear layer length normalized with bubble length for various angles of attack with flap angle being the parameter. 
gradients. Flow separates at the blend point of the leading edge and the flat plate when $\alpha=0^{\circ}$ and $\beta=0^{\circ}$. The onset of separation shifts upstream for increasing values of $\alpha$ and is more pronounced for positive values of $\beta$. The transition location remains almost unchanged for all values of $\beta$ at $\alpha=0^{\circ}$. However, for increasing $\alpha$, the transition point shifts upstream leading to reduction in laminar length of the shear layer. For the cases investigated, the onset of separation occurs between $0.076 C$ and $0.04 C$. Further, the onset of transition shifts from $0.14 C$ to $0.077 C$. A delay of reattachment with angle of attack is clearly evident. Furthermore, a change of pressure gradient by lowering the flap appears to influence the separated layer by moving both transition and reattachment point downstream, but separation point remains almost identical.

The reattachment points $\left(X_{R}\right)$ non-dimensionalised with chord are plotted as a function of angle of attack for $\beta=0^{\circ}$ in figure 10. It shows that the points of reattachment shift downstream with increasing angle of attack and they follow an exponential trend. When the corresponding reattachment points from studies of Arena \& Mueller (1980) are superimposed, they are almost identical. The laminar shear layer length $l_{l}$ is the distance between the point of separation and the onset of transition. Figure 11 shows the comparison of laminar shear layer length normalized with the bubble length plotted as a function of angle of attack. The length of the laminar shear layer falls in the range of $30-61.5 \%$ of the bubble length (that correspond to $11-41 \%$ of the chord) for the cases considered. It is noticed that the percentage length of the laminar shear layer is found to be decreasing with the increasing angles of attack. Figure 12 depicts the bubble length $l_{b}$ (which is a measure from the onset of separation to reattachment) as a function of angle of attack and flap deflections. It illustrates that bubble length increases with angle of attack. Furthermore, the pressure gradient imposed with positive flap angle delays the reattachment as illustrated earlier.

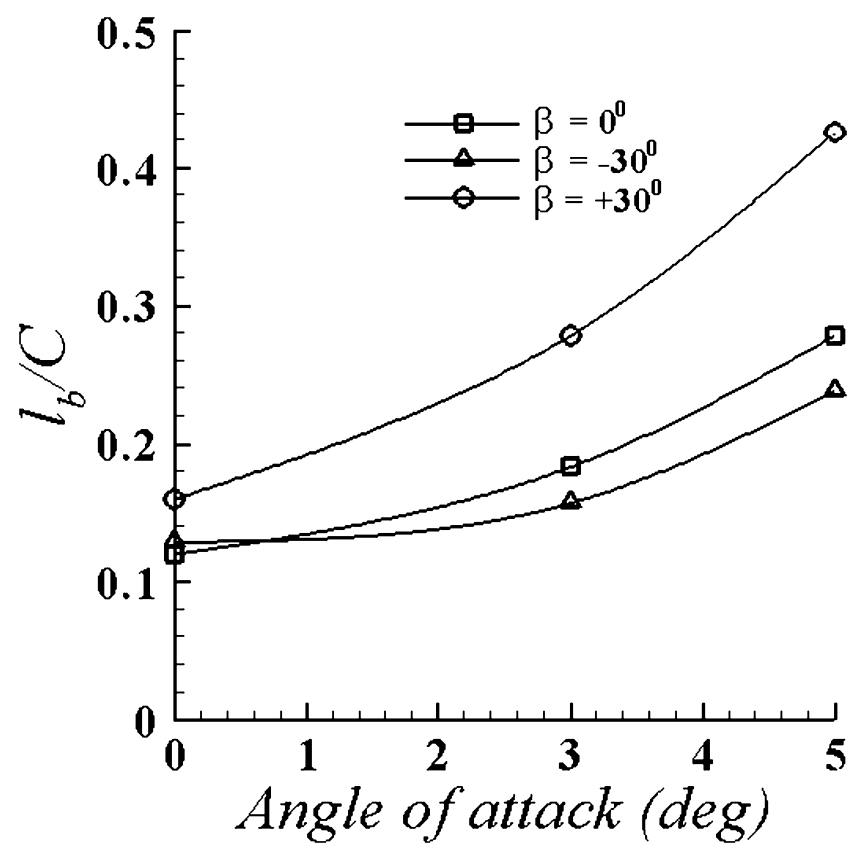

Figure 12. Variation of non-dimensionalised bubble length with respect to chord for various angles of attack subject to tail flap deflections. 
Table 1. Numerical values of pressure recovery coefficient $(\sigma)$ as a function of $\alpha$ for various $\beta$.

\begin{tabular}{lccc}
\hline$\alpha$ & $\beta=0^{\circ}$ & $\beta=-30^{\circ}$ & $\beta=+30^{\circ}$ \\
\hline $0^{\circ}$ & 0.343 & 0.346 & 0.394 \\
$3^{\circ}$ & 0.402 & 0.389 & 0.3504 \\
$5^{\circ}$ & 0.379 & 0.4229 & 0.393 \\
\hline
\end{tabular}

The non-dimensionalised pressure recovery factor $\sigma=\left(C_{p r}-C_{p s}\right) /\left(1-C_{p s}\right)$, is calculated based on the $C_{p}$ values at separation and reattachment. Earlier experimental studies on short bubble (Arena \& Mueller 1980; Crabtree 1969) show that the value of $\sigma$ gradually rises with increasing angles of attack or decreasing Reynolds number for a fixed angle of incidence. Also, it is reported that the short bubble can sustain its identity for a limiting value of $\sigma=0.35$, beyond which the bubble attributes to the flow pattern of a long bubble. For the model of our configuration, it is observed that the bubble remains short for $\alpha=0^{\circ}$ subject to flap angle deflections of $0^{\circ}$ and $-30^{\circ}$ and for the rest other combinations, the bubble remains long as it is evident from the tabulated results in table 1 .

Smoke flow visualizations are taken to obtain a qualitative insight on the overall behaviour of the separated shear layer. Figure 13(a-c) depicts the flow characteristics for three instances when $\alpha=3^{\circ}$ and $\beta=-30^{\circ}$, whereas figure 13(d) shows for $\alpha=7^{\circ}$ and $\beta=-30^{\circ}$. For lower angle of attack $\left(\alpha=3^{\circ}\right)$, flow accelerates over the semi-circular leading edge and it tends to separate from

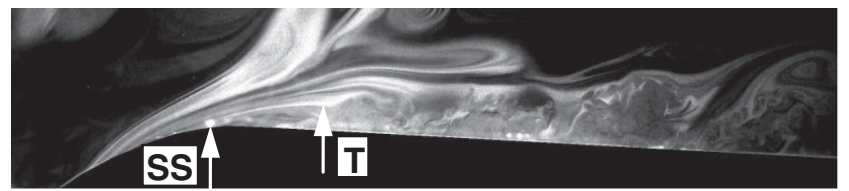

(a) $\beta=-30^{\circ} ; \alpha=3^{\circ}$

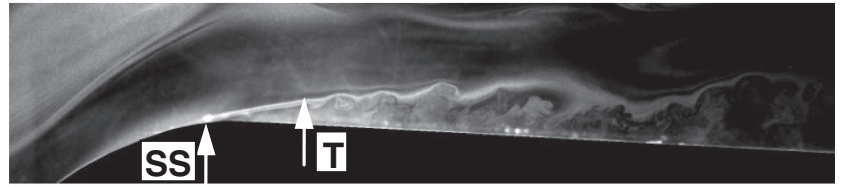

(b) $\beta=-30^{\circ} ; \alpha=3^{\circ}$

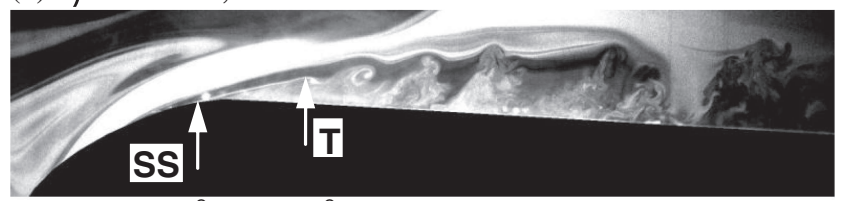

(c) $\beta=-30^{\circ} ; \alpha=3^{\circ}$

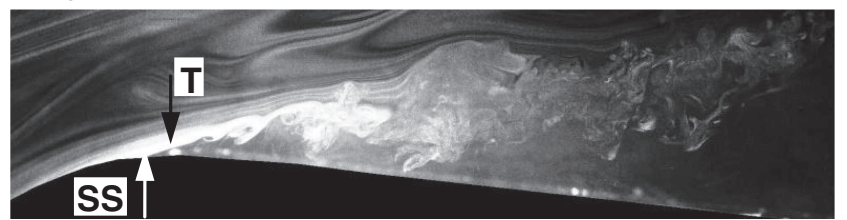

(d) $\beta=-30^{\circ} ; \alpha=7^{\circ}$

SS-Separation; T-Transition

Figure 13. Instantaneous smoke flow visualization pictures taken at $R e_{c}=2.44 \times 10^{5}$. 
the blend point. The shear layer is laminar in the beginning. The instability of the separated layer occurs because of enhanced receptivity of perturbations with appearance of deformation in the smoke streak line that initiates $(K-H)$ rolls at approximately $25 \%$ of bubble length. These $K-H$ rolls convect, grow and become three-dimensional, shedding large-scale vortices that keep their identity far downstream. It is noticed that transition might happen over a region, as it is evinced from the instantaneous pictures, where the laminar length of the shear layer grows and falls with time in the streamwise direction (Arena \& Mueller 1980). For higher angles of attack, shear layer is lifted off and fails to reattach depicting bubble bursting, figure 13(d).

To quantify the shear layer, streamwise velocity contours superimposed with vectors obtained from PIV measurement are shown in figure 14 for $\alpha=0^{\circ}$ and $\beta= \pm 30^{\circ}$. The mean bubble structure shown in figure 14(a) and 14(d) is obtained by averaging one hundred instantaneous image pairs. Figure 14(b,c) and 14(e,f) illustrates the flow dynamics at two different instants. The flow separates near the blend point and reattaches at $X / C=0.1828$ for $\beta=-30^{\circ}$ and at $X / C=0.2015$ for $\beta=+30^{\circ}$. The development of unsteadiness and three-dimensional motions are prominent in the second half of the bubble with the formation of $K-H$ rolls, shedding large scale vortices that keep their identity far downstream. This vortex shedding process is attributed to large fluctuations of reattachment point and flapping of shear layer (Hain et al 2009; Hu \& Yang 2008; Sarkar 2007, 2008). It is observed that the instantaneous bubble length fluctuates heavily for $\beta=+30^{\circ}$ attributing to larger flapping of shear layer. The maximum change of instantaneous bubble length is observed to be $50 \%$ of mean bubble length. Yang \& Voke (2001) observed about $53 \%$ change of instantaneous bubble length, where the Re was 3450 based on leading edge diameter.
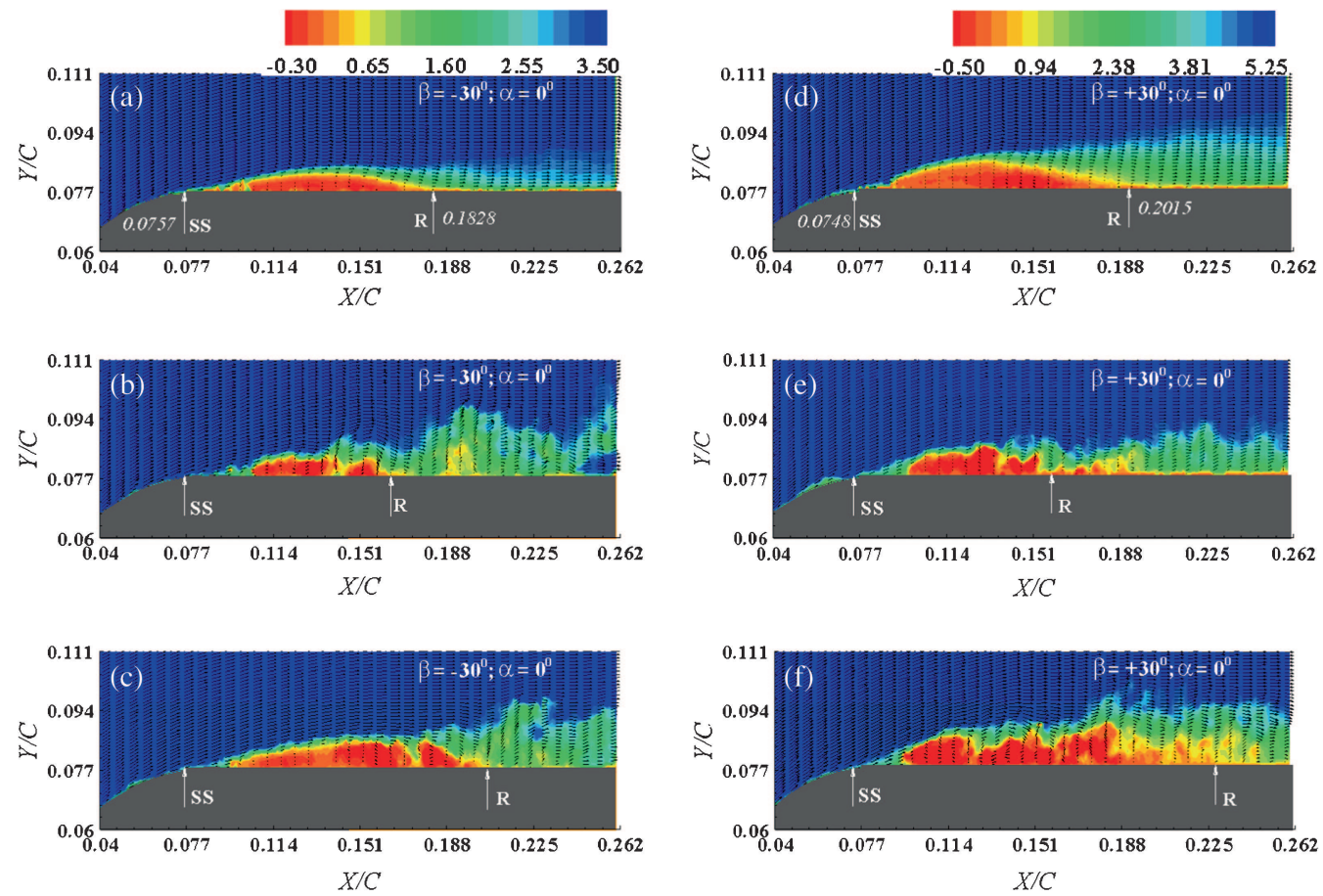

Figure 14. Velocity vectors superimposed with streamwise velocity contours for $\alpha=0^{\circ}$ subject to $\beta= \pm 30^{\circ}$; (a) and (d) averaged flow field, (b, c) and (e, f) instantaneous flow field. 


\section{Conclusions}

Experiments are carried out in an open type blowing tunnel to study the flow characteristics of a laminar separation bubble over a semi-circular leading-edge of a flat plate for varying angles of attack (in the range $\left.0^{\circ}-10^{\circ}\right)$ and flap angle deflections $\left(0^{\circ}\right.$ and $\left.\pm 30^{\circ}\right)$. Positive flap angles affect the shear layer considerably with longer transitional length and delayed reattachment, whereas negative flap angle forces the shear layer to reattach earlier attributing to the formation of smaller bubble. Smoke flow visualization reveals that flow separates near the blending point. The instability of the separated layer occurs because of enhanced receptivity of perturbations with appearance of $\mathrm{K}-\mathrm{H}$ rolls at approximately $25 \%$ of bubble length. The development of unsteadiness and three-dimensional motions are very significant in the second-half of the bubble with shedding of large-scale vortices.

The growth rate of $C_{p}$ is found to be useful in estimating the onset of separation, transition and reattachment point for varying angles of attack and imposed pressure gradients. The onset of separation remains almost identical near to the blending point with a delay of reattachment depicting increase in bubble length, whereas the transition point shifts upstream leading to reduction in laminar length of the shear layer with increase in angle of attack. Furthermore, pressure gradient imposed with positive flap angle deflection appears to influence the separated layer by moving both transition and reattachment point downstream. The onset of transition shifts from $0.14 C$ to $0.077 C$ with increasing $\alpha$, which attributes to the reduction in the laminar shear layer length. The length of the laminar shear layer falls in the range of $30-61.5 \%$ of the bubble length for the cases considered and the percentage length of the laminar shear layer is found to be decreasing with the increasing angles of attack. Considering bubble bursting criteria, it is observed that the bubble remains short for $\alpha=0^{\circ}$ subject to $\beta=0^{\circ}$ and $-30^{\circ}$, whereas the bubble falls in the category of long bubble for all other combinations.

\section{Nomenclature}

$\begin{array}{lll}D & (\mathrm{~mm}) & \text { Leading edge diameter } \\ R e_{c} & (-) & \text { Reynolds number based on chord } \\ C & (\mathrm{~mm}) & \text { Chord length } \\ C_{p} & (-) & \text { Coefficient of pressure }\left(P-P_{r e f}\right) /\left(\frac{1}{2} \rho u_{\infty}^{2}\right) \\ u_{\infty} & (\mathrm{m} / \mathrm{s}) & \text { Free stream velocity } \\ P & \mathrm{~N} / \mathrm{m}^{2} & \text { Surface static pressure } \\ P_{r e f} & \mathrm{~N} / \mathrm{m}^{2} & \text { Reference pressure (Tunnel static pressure }) \\ C_{p}^{\prime} & (-) & \text { Pressure recovery coefficient }\left(C_{p}-C_{p \min }\right) /\left(C_{p \max }-C_{p \min }\right) \\ S S & (-) & \text { Separation onset } \\ T & (-) & \text { Onset of transition } \\ R & (-) & \text { Reattachment location } \\ \alpha & (\mathrm{deg}) & \text { Angle of attack } \\ \beta & (\mathrm{deg}) & \text { Flap deflection } \\ S & (\mathrm{~mm}) & \text { Surface length } \\ X_{R} & (\mathrm{~mm}) & \text { Reattachment length } \\ l_{b} & (\mathrm{~mm}) & \text { Bubble length } \\ l_{l} & (\mathrm{~mm}) & \text { Laminar shear layer length } \\ \sigma & (-) & \text { Pressure recovery factor } \\ T u & (-) & \text { Turbulence Intensity } \\ t & (\mathrm{~s}) & \text { Time }\end{array}$




\section{Subscripts}

$\begin{array}{ll}\min & \text { Minimum } \\ \max & \text { Maximum } \\ s & \text { Separation } \\ r & \text { Reattachment }\end{array}$

\section{References}

Arena A V and Mueller T J 1980 Laminar separation, transition and turbulent reattachment near the leading edge of airfoils. AIAA J. 18(7): 747-753

Boutilier M S H and Yarusevych S 2012 Parametric studies of separation and transition characteristics over an airfoil at low Reynolds numbers. Exp. Fluids 52: 1491-1506

Cherry N J, Hillier R and Latour M E M P 1983 The unsteady structure of two-dimensional separated and reattaching flows. J. Wind Eng. Ind. Aero. 11: 95-105

Crabtree L F 1969 The formation of regions of separated flow on wing surfaces. A.R.C. Technical Report, R. \& M. No. 3122

Gaster M 1967 The structure and behaviour of laminar separation bubbles. Aerodynamics Division N.P.L., Reports and Memoranda No. 3595

Gerakopulos R, Boutilier M S H and Yarusevych S 2010 Aerodynamic characterization of a NACA 0018 airfoil at low Reynolds numbers. Proceedings of the Fluid Dynamics Conference and Exhibit, AIAA 2010-4629

Hain R, Khaler C J and Radespiel R 2009 Dynamics of laminar separation bubbles at low-Reynolds-number aerofoils. J. Fluid Mech. 630: 129-153

Horton H P 1968 Laminar separation bubbles in two and three-dimensional incompressible flow. Ph.D. thesis, Department of Aeronautical Engineering, Queen Mary College, University of London. C. P. No., 1073

$\mathrm{Hu} \mathrm{H}$ and Yang Z 2008 An experimental study of the laminar flow separation on a low-Reynolds-number airfoil. J. Fluids Eng. 130: 051101-11

Jones B M 1934 Stalling. J. R. Aero. Soc. 38: 747-770

Lin J C M and Pauley L L 1996 Low-Reynolds-number separation on an airfoil. AIAA J. 34(8): 1570-1577

McAuliffe B R and Yaras M I 2005 Separation-bubble-transition measurements on a low-Re airfoil using particle image velocimetry. Proceedings of ASME Turbo Expo, Paper No. GT2005-68663

O’Meara M M and Mueller T J 1987 Laminar separation bubble characteristics on an airfoil at low Reynolds numbers. AIAA J. 25(8): 1033-1041

Roskho A and Lau J C 1965 Some observations on transition and reattachment of a free shear layer in incompressible flow. Proceedings of the Heat Transfer and Fluid Mechanics Institute, Stanford University Press, 157-167

Samson A, Sarkar S and Anand K 2012 Experimental investigation of a separation bubble on a flat plate with semi-circular leading edge for different Reynolds numbers. International Conference on Heat Transfer, Fluid Mechanics and Thermodynamics, 316-325

Sarkar S 2007 The effects of passing wakes on a separating boundary layer along a low-pressure turbine blade through large eddy simulation. J. Power Energy Proc. Part A IMechE, UK 221: 551-564

Sarkar S 2008 Identification of flow structures on a LP turbine blade due to periodic passing wakes. ASME J. Fluids Eng. 130: 061103

Tani I 1964 Low speed flows involving bubble separations. Progress in aeronautical sciences, Pergamon Press Wahidi R and Bridges D H 2012 Effects of distributed suction on an airfoil at low Reynolds number. AIAA J. 50(3): 523-539

Yang Z and Voke P R 2001 Large-eddy simulation of boundary-layer separation and transition at a change of surface curvature. J. Fluid Mech. 439: 305-333

Yarusevych S, Kawall J G and Sullivan P E 2008 Separated-shear-layer development on an airfoil at low Reynolds numbers. AIAA J. 46(12): 3060-3069 